\title{
Differential Impact of Obesity on CD69 Expression in Individuals with Bipolar Disorder and Healthy Controls
}

\author{
Ana S. Yamagata ${ }^{a}$ Lucas B. Rizzo ${ }^{a}$ Raphael O. Cerqueira ${ }^{a}$ Janine Scott $^{b, c}$ \\ Quirino Cordeiro $^{d}$ Roger S. Mclntyre ${ }^{e}$ Rodrigo B. Mansure Elisa Brietzke ${ }^{\text {a e }}$ \\ ${ }^{a}$ Research Group in Molecular and Behavioral Neuroscience of Bipolar Disorder, Department of Psychiatry, Federal \\ University of São Paulo, São Paulo, Brazil; ' Institute of Neuroscience, Newcastle University, Newcastle upon Tyne, \\ UK; ' Centre for Affective Disorders, loPPN, Kings College, London, UK; d Psychiatry Department, Santa Casa School of \\ Medical Sciences, São Paulo, Brazil; ' Mood Disorders Psychopharmacology Unit (MDPU), University Health Network, \\ Toronto, ON, Canada
}

\section{Keywords}

Bipolar disorder · Immunosenescence · Inflammation .

Obesity $\cdot \mathrm{CD} 69 \cdot \mathrm{T}$ cell activation

\begin{abstract}
Preliminary evidence suggests that premature immunosenescence is involved in bipolar disorder (BD) pathophysiology. The cellular marker CD69 is expressed in T lymphocyte surface during their activation and its expression is negatively correlated with age. The objective of this study was to assess the moderating effects of obesity on the reduction of expression of CD69, a marker of immunosenescence. Forty euthymic patients with BD type I, aged 18-65 years, were included in this study. The healthy comparison group consisted of 39 volunteers who had no current or lifetime history of mental disorders, no use of psychotropic medications, and no known family history of mood disorders or psychosis. Peripheral blood mononuclear cells from BD patients and healthy controls were collected and isolated. The cells were allowed to grow in culture and stimulated for 3 days. CD69 was marked and read in flow cytometry. We found that
\end{abstract}

the lower expression of CD69 in BD patients was moderated by body mass index (BMI) in both $\mathrm{CD} 4+(\mathrm{RR}=0.977,95 \% \mathrm{Cl}$ $0.960-0.995, p=0.013)$ and CD8+ cells $(\mathrm{RR}=0.972,95 \% \mathrm{Cl}$ $0.954-0.990, p=0.003)$. Our findings indicate that BMI could potentially influence the process of premature aging in $\mathrm{BD}$.

(c) 2018 S. Karger AG, Basel

\section{Introduction}

The presence of immune-inflammatory dysfunctions in bipolar disorder (BD) has been robustly documented in several cross-sectional studies $[1,2]$. However, significant controversies remain about causes, consequences and possible confounders of the abnormal interactions between the brain and the immune system in this disorder [3]. Recently, our group and others have proposed the paradigm of premature aging as a theoretical framework to integrate replicated findings on imbalance in oxidative stress control, telomere shortening, and persistent lowgrade inflammation, all of them extensively documented during normative aging as well as in $\mathrm{BD}$ [4-7].

\section{KARGER}

(c) 2018 S. Karger AG, Basel

E-Mail karger@karger.com

www.karger.com/mnp 
Table 1. Clinical and demographic characteristics of the sample

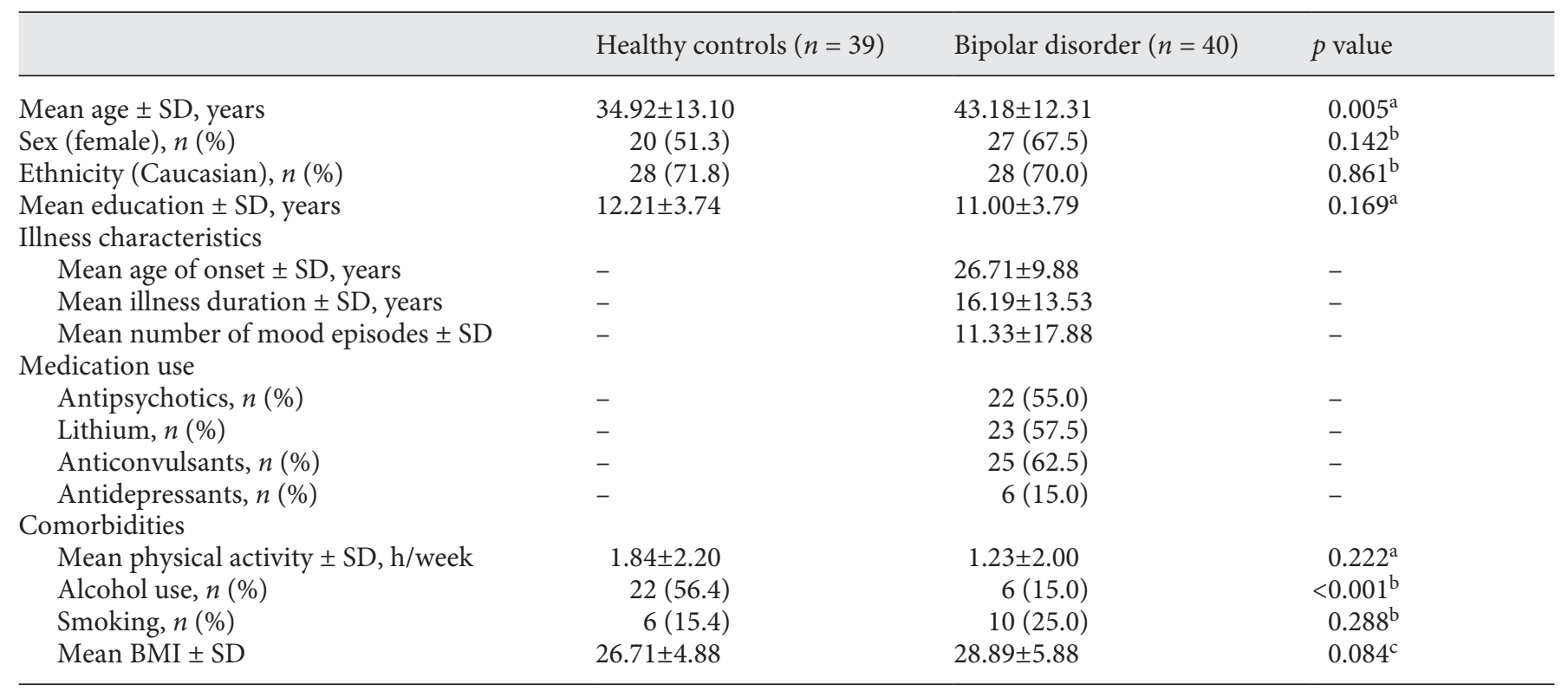

SD, standard deviation; BMI, body mass index. ${ }^{\mathrm{a}} t$ test. ${ }^{\mathrm{b}} \chi^{2}$ test. ${ }^{\mathrm{c}}$ Mann-Whitney test.

Regarding immune-inflammatory changes, $\mathrm{BD}$ has been associated with an increase in pro-inflammatory cytokines [8] and the rate of senescent T cells [4]. Data from do Prado et al. [9] showed that individuals with BD prematurely present markers of senescence of $\mathrm{T}$ cells and an increased proportion of regulatory $\mathrm{T}$ cells, a profile similar to what is described in the elderly. Among the several factors that are able to influence immune function in $\mathrm{BD}$, obesity has received relatively little attention. Nevertheless, the impact of obesity in the clinical course of $\mathrm{BD}$ is well documented. Obese individuals with $\mathrm{BD}$ have a higher frequency of severe presentations compared to normal-weight patients, including rapid cycling [10], depressive relapse [11], cognitive impairments [12-14], suicidality, and comorbidity with substance abuse disorders [15]. In addition, overweight and obesity, especially in case of abdominal fat accumulation, are associated with systemic low-grade inflammation, and this association could be responsible for the myriad of metabolic complications related to obesity, such as glucose changes [16]. Nevertheless, the influence of obesity on premature immunosenescence in $\mathrm{BD}$ has not, to our knowledge, been previously evaluated.

The cellular marker CD69 in T lymphocytes is expressed in the beginning of their activation $[17,18]$, and its increased expression (which is evoked by an immune challenge) is attenuated with aging. This finding is com- monly interpreted as an age-related reduction in the efficiency of the proliferative response [19]. Although few studies have evaluated CD69 expression in obese individuals, CD69 expression in response to vaccination (i.e., an immune challenge) was shown to be lower in individuals who were overweight, compared to those of normal weight [20], suggesting a reduced proliferative response associated with obesity.

We therefore hypothesized that obesity moderates the expression of CD69 in T lymphocytes in individuals with $\mathrm{BD}$. The objective of the present study was to compare the impact of body mass index (BMI) on the CD69 expression in $\mathrm{T}$ cells in culture from individuals with $\mathrm{BD}$ and healthy controls (HC).

\section{Methods}

The research protocol for this study was approved by the local Ethics Committee and all participants provided written informed consent prior to study inclusion.

\section{Studied Population}

Forty male and female euthymic patients with BD type I according to DSM-IV criteria, aged 18-65 years, were included. Diagnosis was confirmed using the Structured Clinical Interview for DSM-IV Axis 1, conducted by a trained psychiatrist. Mania and severity of depression symptoms were assessed using the Young Mania Rating Scale and the 17-item Hamilton Depression Rating 


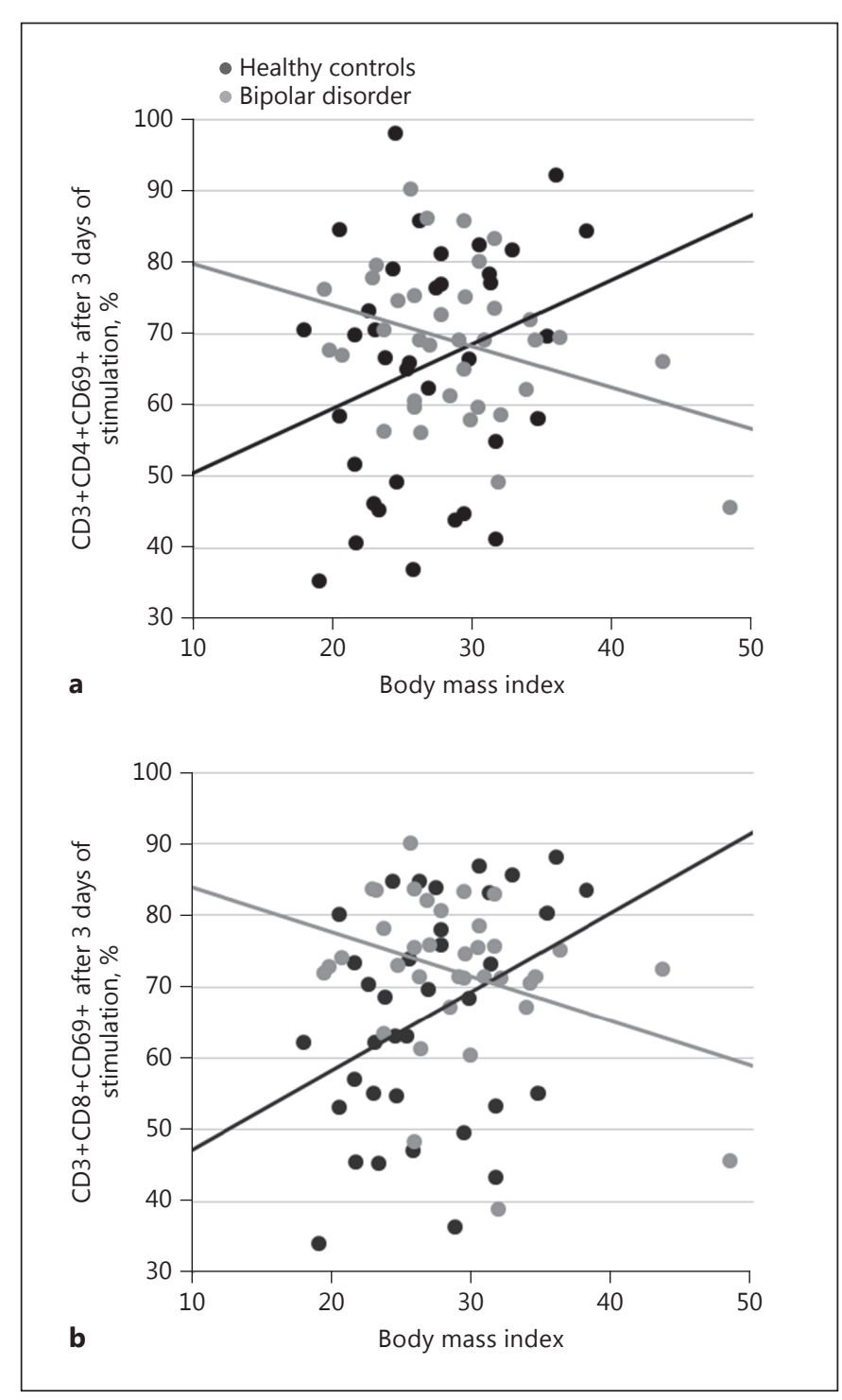

Fig. 1. Association between body mass index and CD69 expression in CD4 (a) and CD8 (b) T cells on the third day after in vitro stimulation, from healthy controls and individuals with bipolar disorder.

Scale, respectively. Euthymia was defined as not fulfilling DSM-IV criteria for mood episode and not having Hamilton Depression Rating Scale and Young Mania Rating Scale scores higher than 8. Only individuals with a stable medication regimen (defined as taking the same medications in the same doses for a minimum of 2 months prior to the study inclusion) were included. The healthy comparison group (HC) consisted of 39 volunteers who had no current or lifetime history of mental disorders or use of psychotropic medications and without a family history of mood disorders, psychosis or suicide in first-degree relatives. The exclusion criteria for both groups were: recent use of anti-inflammatory medications (up to and including the previous 2 weeks); long-term treatment with immunomodulatory medications; acute or chronic general medical conditions related to immune dysfunction or chronic inflammation; and pregnancy or breastfeeding.

\section{CD69 Assessment}

Approximately $15 \mathrm{~mL}$ of venous blood was withdrawn in EDTA tubes between 8 and 10 a.m. after a 12-h period of fasting. The plasma was isolated by centrifugation and frozen at $-80^{\circ} \mathrm{C}$. Peripheral blood mononuclear cells (PBMCs) were isolated by density gradient using Ficoll. Ninety-six-well plates were coated with $5 \mu \mathrm{g} / \mathrm{mL}$ of anti-human CD3 antibody (OKT3, eBioscience) overnight at $4^{\circ} \mathrm{C}$. Then, $1 \times 10^{5}$ PBMCs were added to the wells together with anti-human CD28 antibody (CD28.2, eBioscience) at a final concentration of $5 \mu \mathrm{g} / \mathrm{mL}$. PBMCs were cultivated in RPMI-1640 culture medium supplemented with L-glutamine, $10 \%$ fetal bovine serum, antibiotics, and antimycotics (all from LifeThermo Fischer). The cells were allowed to grow for 3 days at $37^{\circ} \mathrm{C}$ in a $5 \% \mathrm{CO}_{2}$ atmosphere. The experiment always included a negative control (PBMC without anti-CD3 and anti-CD28 cultivated for 3 days). On day 3 , the T helper and T cytotoxic activation marker CD69 was counted and stained. Cells were analyzed by flow cytometry.

\section{Statistics}

All analyses were undertaken in IBM SPSS version 24.0. For comparison of sociodemographic data, the $\chi^{2}$ and $t$ tests were used. As the distribution of CD69 expression was positively skewed, a generalized linear model with a gamma distribution and a log-link function were used to analyze the main effects and interactions of group (BD vs. HC) and BMI, as well as of covariates (i.e., age and gender). To examine proliferative capacity, generalized estimating equation models for repeated measures were used. Time ( 0 vs. 3 days) by group interactions considering each immunological variable were analyzed. Due to the nonlinearity of the models, the estimated $\beta$ coefficients were transformed into RR estimates. The significance level was set at $p<0.05$.

\section{Results}

Clinical and demographic data of the studied population are shown in Table 1. Compared to HC, individuals with BD were significantly older. We did not observe any intergroup differences in the proportion of males and females or in the proportion of Caucasians. There were no statistically significant differences between groups in BMI, number of hours of physical exercise per week, or in tobacco smoking habits.

The expression of CD69 on the third day after stimulation in culture was moderated by BMI, for both CD4 $(\mathrm{RR}=0.981,95 \%$ CI $0.964-0.997, p=0.024)$ and CD8 $(\mathrm{RR}=0.976,95 \%$ CI $0.959-0.992, p=0.004)$ lymphocyte subpopulations (Fig. 1). Further adjustment for physical activity, medications and use of alcohol or tobacco did not modify the results (CD4: $\mathrm{RR}=0.981,95 \%$ CI $0.964-$ $0.998, p=0.027$; CD8: $\mathrm{RR}=0.981,95 \%$ CI $0.965-0.998$, 
$p=0.026)$. Longitudinal analysis showed that percentage changes in CD69 expression was moderated by a group by BMI interaction for both CD4 (RR $=0.977,95 \% \mathrm{CI}$ $0.960-0.995, p=0.013)$ and CD8 cells $(\mathrm{RR}=0.972,95 \%$ CI 0.954-0.990, $p=0.003$ ).

\section{Discussion}

Results from this study indicated that the reduction of expression of CD69 on the third day after stimulation in culture, a marker of immunosenescence, was moderated by BMI, for both CD4 and CD8 lymphocyte subpopulations extracted from peripheral blood of individuals with mood disorders. Further adjustment for physical activity, medications, and use of alcohol or tobacco did not change the results indicating that this effect was not due to possible confounders commonly found in this population. Longitudinal analysis showed that percentage changes in CD69 expression was moderated by a group by BMI interaction for both CD4 and CD 8 cells. These findings suggest that the decrease in CD69 expression in CD4 and CD8 T cells from BD individuals, compared to $\mathrm{HC}$, was indeed mediated by BMI, suggesting that $\mathrm{BD}$ and $\mathrm{BMI}$ have a synergistic effect on $\mathrm{T}$ cell senescence.

Although the deleterious impact of overweight/obesity on the clinical course of BD is well known, few studies evaluated the mechanisms involved in this association [21]. Only recently were immune dysfunctions started to be hypothesized as a link between obesity and a severe course of $\mathrm{BD}$ [22]. It is possible that chronic activation of the immune system associated with obesity may be related to decline in $\mathrm{T}$ cell function including signal transduction, IL-2 expression, and proliferation [23]. Although specific mechanisms for the effect of obesity on immunosenescence still remain largely speculative, the adipose tissue-derived hormone leptin has been pointed out as a contributor, as its concentration was associated with immunosenescence markers [11, 12, 24, 25]. In addition, the state of "inflammaging" in obese individuals with $\mathrm{BD}$ may be partially explained by the excessive free fatty acids that activate toll-like receptor 4 leading to increased release of inflammatory cytokines, pro-inflammatory macrophage infiltration in the adipose tissue, gut microbiota changes, and other mechanisms involved in obesity-induced inflammation [26]. Additionally, chronic stress, present in BD with longstanding hypothalamuspituitary-adrenal axis hyperactivity, could also be a contributor to such an association [27].

Differential Impact of Obesity on CD69 Expression in $\mathrm{BD}$ and Healthy Controls
The results of this study should be interpreted in light of some limitations. The small sample size including only euthymic individuals precluded subgroup analysis as well as evaluation of the effect of different mood states. Nonetheless, other parameters of immunosenescence need to be examined with BMI to further develop the rationale. The cross-sectional design did not allow conclusions about the cause-effect relationship and the effect of medications was also not assessed.

The current study is an attempt to offer new insights into the complex influence of BMI on the immune dysregulation of $\mathrm{BD}$ and to suggest that future studies should carefully include, in their outcome evaluations, the influence of obesity on peripheral levels of immune mediators. In addition, it should be highlighted that some relevant immune markers cannot be assessed in a single peripheral measurement and will need more sophisticated methodologies of analysis, such as cell culture.

This study was the first to demonstrate a possible moderational effect of BMI on the reduction of expression of CD69 after stimulation in culture from individuals with $\mathrm{BD}$ type 1 . This suggests the possibility of participation of obesity in reducing $\mathrm{T}$ cell activation and proliferation in individuals with $\mathrm{BD}$.

\section{Acknowledgment}

The study was funded by CNPq, Brazil.

\section{Statement of Ethics}

The protocol was approved by the Ethics Research Board and all subjects provided written informed consent prior to their inclusion.

\section{Disclosure Statement}

The authors declare that they have no conflicts of interest.

\begin{tabular}{|c|c|}
\hline References & $\begin{array}{l}1 \text { Modabbernia A, Taslimi S, Brietzke E, Ashrafi } \\
\text { M: Cytokine alterations in bipolar disorder: a } \\
\text { meta-analysis of } 30 \text { studies. Biol Psychiatry } \\
\text { 2013;74:15-25. } \\
2 \text { Brietzke E, Stabellini R, Grassi-Oliveira R, } \\
\text { Lafer B: Cytokines in bipolar disorder: recent } \\
\text { findings, deleterious effects but promise for } \\
\text { future therapeutics. CNS Spectr 2011;16:157- } \\
\text { 168. } \\
3 \text { Rosenblat JD, McIntyre RS: Bipolar disorder } \\
\text { and inflammation. Psychiatr Clin North Am } \\
\text { 2016;39:125-137. }\end{array}$ \\
\hline
\end{tabular}


4 Rizzo LB, Costa LG, Mansur RB, Swardfager W, Belangero SI, Grassi-Oliveira R, McIntyre RS, Bauer ME, Brietzke E: The theory of bipolar disorder as an illness of accelerated aging: implications for clinical care and research. Neurosci Biobehav Rev 2014;42:157-169.

5 Rizzo LB, Do Prado CH, Grassi-Oliveira R, Wieck A, Correa BL, Teixeira AL, Bauer ME: Immunosenescence is associated with human cytomegalovirus and shortened telomeres in type I bipolar disorder. Bipolar Disord 2013; 15:832-838.

6 Squassina A, Pisanu C, Congiu D, Caria P, Frau D, Niola P, Melis C, Baggiani G, Lopez JP, Cruceanu C, Turecki G, Severino G, Bocchetta A, Vanni R, Chillotti C, Del Zompo M: Leukocyte telomere length positively correlates with duration of lithium treatment in bipolar disorder patients. Eur Neuropsychopharmacol 2016;26:1241-1247.

7 Barbé-Tuana FM, Parisi MM, Panizzutti BS, Fries GR, Grun LK, Guma FT, Kapczinski F, Berk M, Gama CS, Rosa AR: Shortened telomere length in bipolar disorder: a comparison of the early and late stages of disease. Rev Bras Psiquiatr 2016;38:281-286.

8 Brietzke E, Stertz L, Fernandes BS, KauerSant'anna M, Mascarenhas M, Escosteguy Vargas A, Chies JA, Kapczinski F: Comparison of cytokine levels in depressed, manic and euthymic patients with bipolar disorder. J Affect Disord 2009;116:214-217.

9 do Prado CH, Rizzo LB, Wieck A, Lopes RP, Teixeira AL, Grassi-Oliveira R, Bauer ME: Reduced regulatory $\mathrm{T}$ cells are associated with higher levels of Th1/TH17 cytokines and activated MAPK in type 1 bipolar disorder. Psychoneuroendocrinology 2013;38:667-676.

10 Buoli M, Dell'Osso B, Caldiroli A, Carnevali GS, Serati M, Suppes T, Ketter TA, Altamura AC: Obesity and obstetric complications are associated with rapid-cycling in Italian patients with bipolar disorder. J Affect Disord 2017;208:278-283.
11 Bond DJ, Andreazza AC, Hughes J, Dhanoa T, Torres IJ, Kozicky JM, Young LT, Lam RW, Yatham LN: Association of peripheral inflammation with body mass index and depressive relapse in bipolar disorder. Psychoneuroendocrinology 2016;65:76-83.

12 Yim CY, Soczynska JK, Kennedy SH, Woldeyohannes HO, Brietzke E, McIntyre RS: The effect of overweight/obesity on cognitive function in euthymic individuals with bipolar disorder. Eur Psychiatry 2012;27:223-228.

13 Lackner N, Bengesser SA, Birner A, Painold A, Fellendorf FT, Platzer M, Reininghaus B, Weiss EM, Mangge H, McIntyre RS, Fuchs D, Kapfhammer HP, Wallner-Liebmann SJ, Reininghaus EZ: Abdominal obesity is associated with impaired cognitive function in euthymic bipolar individuals. World J Biol Psychiatry 2016;17:535-546.

14 Silveira LE, Kozicky JM, Muralidharan K, Bücker J, Torres IJ, Bond DJ, Kapczinski F, Kauer-Sant'Anna M, Lam RW, Yatham LN: Neurocognitive functioning in overweight and obese patients with bipolar disorder: data from the Systematic Treatment Optimization Program for Early Mania (STOP-EM). Can J Psychiatry 2014;59:639-648.

15 Goldstein BI, Liu SM, Schaffer A, Sala R, Blanco C: Obesity and the three-year longitudinal course of bipolar disorder. Bipolar Disord 2013; 15:284-293.

16 van Greevenbroek MM, Schalkwijk CG, Stehouwer CD: Obesity-associated low-grade inflammation in type 2 diabetes mellitus: causes and consequences. Neth J Med 2013;71:174187.

17 Biselli R, Matricardi PM, D'Amelio R, Fattorossi A: Multiparametric flow cytometric analysis of the kinetics of surface molecule expression after polyclonal activation of human peripheral blood T lymphocytes. Scand J Immunol 1992;35:439-447.

18 Caruso A, Licenziati S, Corulli M, Canaris $A D$, De Francesco MA, Fiorentini S, Peroni L, Fallacara F, Dima F, Balsari A: Flow cytometric analysis of activation markers on stimulated T-cells and their correlation with cell proliferation. Cytometry 1997;27:71-76.
19 Lio D, Candore G, Cigna D, D’Anna C, Di Lorenzo G, Giordano C, Lucania G, Mansueto $\mathrm{P}$, Melluso M, Modica MA: In vitro T cell activation in elderly individuals: failure in CD69 and CD71 expression. Mech Ageing Dev 1996;89:51-58.

20 Sheridan PA, Paich HA, Handy J, Karlsson EA, Hudgens MG, Sammon AB, Holland LA Weir S, Noah TL, Beck MA: Obesity is associated with impaired immune response to influenza vaccination in humans. Int J Obes 2012; 36:1072-1077.

21 Liu CS, Carvalho AF, Mansur RB, McIntyre RS: Obesity and bipolar disorder: synergistic neurotoxic effects? Adv Ther 2013;30:9871006.

22 Yamagata AS, Mansur RB, Rizzo LB, Rosenstock T, McIntyre RS, Brietzke E: Selfish brain and selfish immune system interplay: a theoretical framework for metabolic comorbidities of mood disorders. Neurosci Biobehav Rev 2017;72:43-49.

23 Huang H, Patel DD, Manton KG: The immune system in aging: roles of cytokines, T cells and NK cells. Front Biosci 2005;10:192-215.

24 Sanchez-Margalet V, Martin-Romero C, Santos-Alvarez J, Goberna R, Najib S, GonzalezYanes C: Role of leptin as an immunomodulator of blood mononuclear cells: mechanisms of action. Clin Exp Immunol 2003;133:11-19.

25 Ovsyannikova IG, White SJ, Larrabee BR, Grill DE, Jacobson RM, Poland GA: Leptin and leptin-related gene polymorphisms, obesity, and influenza $\mathrm{A} / \mathrm{H} 1 \mathrm{~N} 1$ vaccine-induced immune responses in older individuals. Vaccine 2014;32:881-887.

26 Gregor MF, Hotamisligil GS: Inflammatory mechanisms in obesity. Annu Rev Immunol 2011;29:415-445.

27 Soczynska JK, Kennedy SH, Woldeyohannes HO, Liauw SS, Alsuwaidan M, Yim CY, McIntyre RS: Mood disorders and obesity: understanding inflammation as a pathophysiological nexus. Neuromolecular Med 2011;13: 93-116. 